\title{
The geometrical origin of dark energy
}

\author{
Alon E. Faraggi ${ }^{1}$, Marco Matone M $^{2,3, \mathrm{a}}$ (i) \\ ${ }^{1}$ Department of Mathematical Sciences, University of Liverpool, Liverpool L69 7ZL, UK \\ 2 Dipartimento di Fisica e Astronomia "G. Galilei”, Università di Padova, Via Marzolo 8, 35131 Padua, Italy \\ ${ }^{3}$ INFN, Sezione di Padova, Via Marzolo 8, 35131 Padua, Italy
}

Received: 11 October 2020 / Accepted: 13 November 2020 / Published online: 27 November 2020

(C) The Author(s) 2020

\begin{abstract}
The geometrical formulation of the quantum Hamilton-Jacobi theory shows that the quantum potential is never trivial, so that it plays the rôle of intrinsic energy. Such a key property selects the Wheeler-DeWitt (WDW) quantum potential $Q\left[g_{j k}\right]$ as the natural candidate for the dark energy. This leads to the WDW Hamilton-Jacobi equation with a vanishing kinetic term, and with the identification

$\Lambda=-\frac{\kappa^{2}}{\sqrt{\bar{g}}} Q\left[g_{j k}\right]$.

This shows that the cosmological constant is a quantum correction of the Einstein tensor, reminiscent of the von Weizsäcker correction to the kinetic term of the ThomasFermi theory. The quantum potential also defines the Madelung pressure tensor. The geometrical origin of the vacuum energy density, a strictly non-perturbative phenomenon, provides strong evidence that it is due to a graviton condensate. Time independence of the regularized WDW equation suggests that the ratio between the Planck length and the Hubble radius may be a time constant, providing an infrared/ultraviolet duality. We speculate that such a duality is related to the local to global geometry theorems for constant curvatures, showing that understanding the universe geometry is crucial for a formulation of Quantum Gravity.
\end{abstract}

\section{Introduction}

In spite of the tremendous efforts, understanding the origin of the cosmological constant [1-3] is still an open question. In this paper we show that the cosmological constant is naturally interpreted in terms of the quantum potential associated to the spatial metric tensor. The starting point concerns the geometrical formulation of the Quantum Hamilton-Jacobi Equation (QHJE), suggested by the $x-\psi$ duality observed in [4] and introduced in [5-10] (see [11] for a short review). In the following we call such a formulation, which differs with respect

a e-mail: matone@pd.infn.it (corresponding author) to the Bohmian one, Geometrical Quantum Hamilton-Jacobi (GQHJ) theory. Such a theory reproduces the main results of Quantum Mechanics (QM), including energy quantization and tunneling, without using any probabilistic interpretation of the wave function, which is one of the problems in formulating a consistent theory of quantum gravity.

Another consequence of the GQHJ theory is that if space is compact, then there is no notion of particle trajectory [12]. It follows that the GQHJ theory reproduces the results of QM following a geometrical approach without the axiomatic interpretation of the wave function as probability amplitude.

The idea underlying the geometrical derivation of the QHJE is that, like General Relativity (GR), even QM has a geometrical interpretation. This is done by imposing the existence of point transformations connecting different states, which, in turn, leads to a cocycle condition that uniquely fixes the QHJE. It is then immediate to show that the QHJE implies the Schrödinger equation. In such a formulation, it has been shown that the quantum Hamilton characteristic function $S$ is non-trivial even in the case of the free particle with vanishing energy. Such a result is deeply related to the solution of Einstein's paradox, discussed later, and concerning the classical limit of bound states in the de Broglie-Bohm theory.

In the present paper we are interested in the fact that, unlike in the de Broglie-Bohm theory, the quantum potential in the GQHJ theory is never trivial [5-10]. This happens even in the case of a free particle with vanishing energy. It is just such a property that led in [13] to the proposal that there is a deep relation between QM and gravity. In particular, it was emphasized that the characteristic property of the quantum potential is its universal nature, which is, like gravity, a property possessed by all forms of matter. Subsequently, the deep relation between gravity and QM was also stressed by Susskind in his GR = QM paper [14] and where it is emphasized that where there is quantum mechanics there is also gravity. An explicit relation between quantum mechanics and 
gravity arises in the case of the free particle with vanishing energy, whose quantum potential includes the Planck length $\ell_{P}=\sqrt{\hbar G / c^{3}}[13]$

$Q(x)=\frac{\hbar^{2}}{4 m}\{S, x\}=-\frac{\hbar^{2}}{2 m} \frac{\ell_{P}^{2}}{\left(x^{2}+\ell_{P}^{2}\right)^{2}}$,

where $\{f, x\}=f^{\prime \prime \prime} / f^{\prime}-\frac{3}{2}\left(f^{\prime \prime} / f^{\prime}\right)^{2}$ is the Schwarzian derivative of $f$. Such a result follows by requiring that, in the case of a free particle of energy $E$, the QHJE consistently reproduces both the $\hbar \rightarrow 0$ and $E \rightarrow 0$ limits. On the other hand, since in the problem there are no scales, one is forced to use universal constants. It turns out that the Planck length is the only candidate satisfying the limit conditions, a result related to the invariance of the quantum potential under Möbius transformations of $S$. Since $E=0$ corresponds to the ground state, it follows that $Q$ can be considered as an intrinsic energy.

The GQHJ theory includes another relation between QM and geometry of the universe. Namely, compactness of space would imply that the energy spectra are quantized [12]. The essential reason is that solutions of the Schrödinger equation should satisfy gluing conditions, so implying a quantized spectra, even in the case of the free particle [12]. This is also connected to the problem of definition of time. To see this, note that while in classical mechanics we have the equivalence between the definition of trajectory given by $p=\nabla S$ and the one following by Jacobi theorem, that is

$p=\vec{\nabla} S \quad \longleftrightarrow \quad t-t_{0}=\frac{\partial S}{\partial E}$,

at the quantum level the two definitions do not coincide. As shown in [12,15-23], trajectories, if any, should be defined by the Jacobi theorem. On the other hand, since a compact universe implies a quantized energy spectra, it follows that in this case the derivative of $S$ with respect to $E$ is ill-defined [12]. ${ }^{1}$ We then have

$$
\begin{aligned}
& \text { Compact Universe } \longrightarrow\left\{E_{n}\right\} \longrightarrow \frac{\partial S}{\partial E} \text { is ill-defined } \\
& \longrightarrow \text { no notion of trajectories. }
\end{aligned}
$$

This leads to a possible relation between the problem of time in GR and the fact that time is not an observable in QM. It should be stressed that in Quantum Field Theory (QFT), even particle's spatial position is represented by parameters, so that, like time, even such a notion does not correspond to an observable.

It is worth mentioning that the GQHJ theory has been inspired by uniformization theory, with the Schrödinger

\footnotetext{
${ }_{1}^{1}$ An alternative to the ill-defined derivative $\partial_{E} S$ is to consider finite differences in the $E-S$ plane. One may easily check that this leads to a heuristic uncertainty relation between $E$ and $t$.
}

equation playing the analogous rôle of the uniformizing equation. In particular, the ratio of two linearly independent solutions of the Schrödinger equation, plays the analogous rôle of the inverse of the uniformizing map. The basic duality, that is the Möbius symmetry, which extends to the QHJE in higher dimension [24], is the defining property of the Schwarzian derivative. Such a duality, that relates small and large scales, and acts like the map between different fundamental domains, is at the heart of the proof of the energy quantization [5-10]. The above connection between compactness of space, discrete spectra and the analogies with uniformization theory, suggests that higher dimensional uniformization theory is related to the geometry of the universe. This would imply that Thurston's geometry [25] is the appropriate framework to describe the Universe. In this context, the 3-torus plays a central rôle.

Besides (1.1), also (1.3) provides a relation between small and large scales. In particular, as in the case of a particle in a ring of radius $R$, that gives $E_{n}=n^{2} \hbar^{2} /\left(2 m R^{2}\right), n \in \mathbb{Z}$, an analogous relation shows that the energy spacing depends on the parameters defining the compact geometry of space.

We saw that the GQHJ theory indicates that QM and GR are deeply related. In particular, in the GQHJ theory, time is not a well-defined observable. On the other hand, in the quantum gravity equation par excellence, that is the WheelerDeWitt (WDW) equation [26,27], there is no time variable at all.

The above analysis suggests considering the rôle of the WDW quantum potential. In the case of quantum gravity, the quantum potential represents an intrinsic energy density. In analogy with the GQHJ theory and, in particular, with (1.1), the natural interpretation is that the WDW quantum potential in the vacuum is the one of dark energy, that is

$\Lambda=-\frac{\kappa^{2}}{\sqrt{\bar{g}}} Q\left[g_{j k}\right]$

where $\bar{g}=\operatorname{det} g_{j k}$. We then have that the cosmological constant is a quantum correction to the Einstein tensor. This is reminiscent of the von Weizsäcker correction to the kinetic term of the Thomas-Fermi theory [28]. It is worth mentioning that also the Madelung pressure tensor is defined in terms of the quantum potential.

Since (1.4) refers to the vacuum, it follows that there are no dynamical degrees of freedom, so that $S=0$. This means that (1.4) coincides with the WDW equation in the vacuum.

A consequence of our investigation is that since the metric tensor is the only field involved in (1.4), it follows that dark energy is naturally identified with a graviton condensate. We note that, in a quite different context, the rôle of the (Bohmian) quantum potential in cosmology, suggesting that the vacuum is a graviton condensate, has been proposed in [29]. 
We will argue that, as suggested by Feng's volume average regularization [30], and by the minisuperspace approximation, a regularized WDW equation would need, besides the Planck length, the addition of an infrared scale that we identify with the Hubble radius $R_{H}=c / H_{0}=1.36 \times 10^{26} \mathrm{~m}$. Time independence of the regularized WDW equation would then imply that, like $R_{H}$, even the Planck length is timedependent. In particular, time independence of the WDW wave-functional suggests that

$\mathcal{K}=\frac{\ell_{P}}{R_{H}}=5.96 \times 10^{-61}$,

may be a space-time constant. This would provide an exact infrared/ultraviolet duality.

The paper is organized as follows. In Sect. 2 we shortly review the derivation of the WDW Hamilton-Jacobi (HJ) equation. Section 3 illustrates the main points of the GQHJ theory formulated in [5-10], focusing on its geometrical origin and on the solution of Einstein's paradox, which in turn is related to the non-triviality of the QHJE for the free particle with $E=0$. In Sect. 4 we show that, contrary to the de Broglie-Bohm formulation, the quantum potential is nontrivial even in the case of the WDW HJ equation with ${ }^{3} R=0$ and vanishing cosmological constant. In Sect. 5 we show that the cosmological constant is naturally interpreted in terms of the WDW quantum potential in the vacuum. We then derive the wave-functional in the minisuperspace approximation. Section 6 is devoted to some speculative suggestion relating the infrared/ultravilet duality, in the context of the regularized WDW equation, to the local to global geometry theorems concerning manifolds of constant curvature. It turns out that the global geometry is strongly constrained in case the local one has constant curvature. This is just the geometrical counterpart of the fact that large scale physics seems constrained by the physics at small scales. Another manifestation of the connection between QM and GR. Finally, we argue that time independence of the regularized WDW equation would imply that $\mathcal{K}$ is a space-time constant.

\section{WDW Hamilton-Jacobi equation}

In the Arnowitt, Deser and Misner (ADM) formulation [31], the space-time is foliated into a family of closed space-like hypersurfaces parametrized by time. One then considers such spatial hypersurfaces at "constant time", as level sets of a time function

$\Sigma_{t_{0}}=\left\{x^{k} \mid t\left(x^{k}\right)=t_{0}\right\}$.

In the following we choose the metric signature $(-,+,+,+)$. Denote by $g_{i j}={ }^{4} g_{i j}$ the metric tensor of the three dimensional spatial slices. Let $N=\left(-{ }^{4} g^{00}\right)^{-1 / 2}$ be the lapse and $N_{k}={ }^{4} g_{0 k}$ the shift vector field. We then have the standard
3+1 decomposition

$d s^{2}=\left(N_{k} N^{k}-N^{2}\right) c^{2} d t^{2}+2 N_{k} c d x^{k} d t+g_{j k} d x^{j} d x^{k}$.

Note that $N, N_{k}$ and $g_{j k}$ depend on $\left(t_{0}, x^{1}, x^{2}, x^{3}\right)$. As we will see, the lapse function and the shift vector field play the role of four Lagrange multipliers and describe the welding of the $\Sigma_{t}$ 's. The equations of motion for $N$ and $N_{k}$ are arbitrary, reflecting the freedom in choosing the space-time coordinates [31-33].

Set $\bar{g}=\operatorname{det} g_{j k}$ and $\kappa^{2}=8 \pi G / c^{4}$. The Einstein-Hilbert Lagrangian density can be equivalently expressed in the form

$\mathscr{L}=\frac{1}{2 \kappa^{2}} N \sqrt{\bar{g}}\left({ }^{3} R-2 \Lambda+K^{j k} K_{j k}-K^{2}\right)$,

where ${ }^{3} R$ is the intrinsic spatial scalar curvature, $\Lambda$ the cosmological constant, $K$ the trace of the extrinsic curvature

$K_{j k}=\frac{1}{N}\left(\frac{1}{2} g_{j k, 0}-D_{(j} N_{k)}\right)$,

and $D_{j}$ denotes the $j$ component of the covariant derivative. Let $\pi^{0}$ and $\pi^{k}$ be the momenta conjugate to $N$ and $N_{k}$ respectively. Since $\mathscr{L}$ is independent of both $\partial_{x_{0}} N$ and $\partial_{x_{0}} N_{k}$, we have the primary constraints $\pi^{0} \approx 0, \pi^{k} \approx 0$. Here the symbol " $\approx$ " indicates weak equality, that is the vanishing holding only on the sub-manifold of the phase space constrained by the primary constraints. The equality holding only when the expression is identically vanishing on the full phase space [33].

Time conservation of the primary constraints implies secondary constraints, given by the weak vanishing of the supermomentum,

$\mathcal{H}_{k}=-2 D_{j} \pi^{j}{ }_{k} \approx 0$

and of the super-Hamiltonian,

$\mathcal{H}=2 \kappa^{2} G_{i j k l} \pi^{i j} \pi^{k l}-\frac{1}{2 \kappa^{2}} \sqrt{\bar{g}}\left({ }^{3} R-2 \Lambda\right) \approx 0$,

where $\pi^{j k}$ is the momentum canonically conjugated to $g_{j k}$, that is

$\pi^{j k}=-\frac{1}{2 \kappa^{2}} \sqrt{\bar{g}}\left(K^{j k}-g^{j k} K\right)$,

and

$G_{i j k l}=\frac{1}{2 \sqrt{g}}\left(g_{i k} g_{j l}+g_{i l} g_{j k}-g_{i j} g_{k l}\right)$,

is the DeWitt supermetric. The conservation in time of the secondary constraints do not imply further constraints.

By a Legendre transform one gets the Hamiltonian

$H=\int d^{3} \mathbf{x}\left(N \mathcal{H}+N^{k} \mathcal{H}_{k}\right)$, 
showing that $N$ and $N^{k}$ are the Lagrange multipliers of $\mathcal{H}$ and $\mathcal{H}_{k}$ respectively.

Let $\Psi$ be the Schrödinger wave-functional, that is $i \hbar \partial_{t} \Psi=$ $\hat{H} \Psi$. Implementation of the primary constraints at the quantum level is obtained by setting

$\hat{\pi}^{0}=-i \hbar \frac{\delta}{\delta N}, \quad \hat{\pi}^{k}=-i \hbar \frac{\delta}{\delta N_{k}}$,

so that

$-i \hbar \frac{\delta \Psi}{\delta N}=0, \quad-i \hbar \frac{\delta \Psi}{\delta N_{k}}=0$,

meaning that $\Psi$ does not depend on any of the non-dynamical variables, that is $\Psi$ depends on $g_{j k}$ only.

At the quantum level the conjugate momenta of a field $\phi$ would correspond to $-i \hbar \delta_{\phi}$, so that, since for the $\delta$ distribution in configuration space we have $\left[\delta^{(3)}\right]=L^{-3}$, it follows that $\left[\delta_{\phi}\right]=[\phi]^{-1} L^{-3}$. On the other hand, by (2.7) we have $\left[\pi_{i j}\right]=M T^{-2}$, which is different from the dimension of the canonical choice of $\hat{\pi}^{j k}$, namely $\left[-i \hbar \delta_{g_{j k}}\right]=$ $M L^{-1} T^{-1}$. We then have

$\hat{\pi}^{j k}=-i \hbar c \frac{\delta}{\delta g_{j k}}$,

which also fixes the normalization of the classical relation

$\pi^{j k}=c \frac{\delta S}{\delta g_{j k}}$,

where $S$ is the functional analogue of Hamilton's characteristic function. By (2.12), the super-momentum constraint reads

$\hat{\mathcal{H}}_{k} \Psi=2 i \hbar c g_{k j} D_{l} \frac{\delta \Psi}{\delta g_{l j}}=0$,

which is satisfied if $\Psi$ is invariant under diffeomorphisms of the hypersurface.

The other secondary constraint, that is $\hat{\mathcal{H}} \Psi=0$, is the WDW equation

$\hbar c\left[-2 \ell_{P}^{2} G_{i j k l} \frac{\delta^{2}}{\delta g_{i j} \delta g_{k l}}-\frac{1}{2 \ell_{P}^{2}} \sqrt{\bar{g}}\left({ }^{3} R-2 \Lambda\right)\right] \Psi\left[g_{i j}\right]=0$,

where $\ell_{P}=\sqrt{8 \pi \hbar G / c^{3}}=\kappa \sqrt{\hbar c}$ is the rationalized Planck length. Note that the secondary constraints imply $\hat{H} \Psi=0$, so that $\partial_{t} \Psi=0$, which is the origin of the problem of time.

Let us now consider the key identity

$$
\begin{gathered}
\frac{1}{A \mathrm{e}^{\beta S}} \frac{\delta^{2}\left(A \mathrm{e}^{\beta S}\right)}{\delta g_{i j} \delta g_{k l}}=\beta^{2} \frac{\delta S}{\delta g_{i j}} \frac{\delta S}{\delta g_{k l}}+\frac{1}{A} \frac{\delta^{2} A}{\delta g_{i j} \delta g_{k l}} \\
+\frac{\beta}{2 A^{2}}\left[\frac{\delta}{\delta g_{i j}}\left(A^{2} \frac{\delta S}{\delta g_{k l}}\right)+\frac{\delta}{\delta g_{k l}}\left(A^{2} \frac{\delta S}{\delta g_{i j}}\right)\right],
\end{gathered}
$$

which holds for any complex constant $\beta$. Set $\beta=i / \hbar$ and

$\Psi=A e^{\frac{i}{\hbar} S}$, with $A$ and $S$ taking real values. In this respect, note that if $A e^{\frac{i}{\hbar} S}$ is a solution, then reality of the WDW operator implies that even $A e^{-\frac{i}{\hbar} S}$. This observation is related to the differences between the Bohmian and the GQHJ formulations discussed later. Replacing $\Psi$ in (2.15) with right hand side of (2.17) gives the WDW HJ equation, corresponding to the following quantum deformation of the HJ equation

$$
\begin{gathered}
2(c \kappa)^{2} G_{i j k l} \frac{\delta S}{\delta g_{i j}} \frac{\delta S}{\delta g_{k l}}-\frac{1}{2 \kappa^{2}} \sqrt{\bar{g}}\left({ }^{3} R-2 \Lambda\right) \\
-2(c \kappa \hbar)^{2} \frac{1}{A} G_{i j k l} \frac{\delta^{2} A}{\delta g_{i j} \delta g_{k l}}=0,
\end{gathered}
$$

together with the continuity equation

$G_{i j k l} \frac{\delta}{\delta g_{i j}}\left(A^{2} \frac{\delta S}{\delta g_{k l}}\right)=0$.

The last term in (2.18), that is

$Q=-2(c \kappa \hbar)^{2} \frac{1}{A} G_{i j k l} \frac{\delta^{2} A}{\delta g_{i j} \delta g_{k l}}$,

is called quantum potential. We note that in the classical limit Eq. (2.18) reduces to the classical case (2.6).

A key difference between the GQHJ formulation and the Bohmian one is that, as in the formulation of QM, the $\Psi$ in (2.17) is not in general identified with the wave-functional of the state of the system, rather it is a general solution of the WDW equation. In the next section, we will see that it is precisely such a characteristic of the GQHJ formulation that, unlike the Bohmian one, implies that

1. there is no Einstein's paradox,

2. there is a basic Möbius symmetry, associated to the Schwarzian equation,

3. energy quantization follows without the need of any interpretation of the wave-function,

4. implies that in compact space there is no notion of particle trajectory.

An explicit example of the difference between the GQHJ and Bohmian formulations associated to the WDW HJ equation is provided in Sect. 4. In particular, we will consider the case ${ }^{3} R=0, \Lambda=0$, so that the WDW equation reduces to the free functional differential equation. While in the Bohmian formulation this would imply

$\Psi=0$,

so giving $A=0, S=0$ and $Q=0$, in the GQHJ theory there are non-trivial solutions. Once again, this shows that, contrary to the Bohmian formulation, the quantum potential in the GQHJ theory is never trivial, so that it plays the rôle of intrinsic energy. 


\section{QHJE and Einstein paradox}

In this section we shortly discuss the main aspects of the GQHJ theory [5-10]. Let us start by recalling Einstein's paradox (see e.g. Ref. [34], pg. 243). This concerns the issue in Bohmian mechanics when considering the classical limit for states described by a wave-function corresponding to Hamiltonian eigenstates of any one-dimensional bound state. Let us then consider a state of definite energy $E$ and denote by $\psi_{E}$ the corresponding wave-function. In this case one can easily show that $\psi_{E} \in L^{2}(\mathbb{R})$ is proportional to a real function. Therefore, if one sets, as in Bohm theory, $\psi_{E}=R e^{\frac{i}{\hbar} S}$, then $S$ is a constant. On the other hand, in the Bohmian formulation, $p=\partial_{x} S$ is identified with the mechanical momentum $m \dot{x}$, so that, quantum mechanically, one would have $p=0$. This would imply that, as in the case of the harmonic oscillator, a quantum particle would be at rest and should start moving in the classical limit, where $S$ and $p$ are non-trivial. In other words, it is clear that it is not possible to get a non-trivial $S$ as the $\hbar \rightarrow 0$ limit of $S=0$.

The resolution of the paradox is that the quantum analogue of $S$ is not necessarily the phase of the wave function. As we will show, this in fact also underlies the WKB approximation that, even if one starts with the identification $\psi=\exp \left(i S_{W K B} / \hbar\right)$, with $S_{W K B}$ complex, then real wave functions are identified with a linear combination of in and out waves. In our formulation, such a choice is not ad hoc as in the WKB approximation, rather it follows from the request that the cocycle condition is always satisfied [5-10]. In particular, note that if $\operatorname{Re}^{\frac{i}{\hbar} S}$ is a solution of the stationary Schrödinger equation (SSE), then, this is also the case of $R e^{-\frac{i}{\hbar} S}$. This is the key to introduce the so-called bipolar decomposition

$\psi_{E}=R\left(A e^{\frac{i}{\hbar} S}+B e^{-\frac{i}{\hbar} S}\right)$,

which is equivalent to say that the most general expression for $S$, and therefore for $R$, is given by

$\operatorname{Re}^{\frac{i}{\hbar} S}=A \psi^{D}+B \psi$

with $\psi^{D}$ and $\psi$ two arbitrary linearly independent solutions of the SSE.

As a result, in the case of a real $\psi_{E}$, the only constraint is just $|A|=|B|$ and one gets a non-trivial $S$ with a well-defined classical limit. Such a solution of Einstein's paradox is a consequence of the GQHJ theory, that excludes in a natural way, and from the very beginning, the existence of states with a constant $S$ [5-10]. The use of the bipolar decomposition was previously discussed by Floyd [15-23].

Later we will see that in the case of the WDW HJ equation, both $S$ and the quantum potential are non-trivial even when ${ }^{3} R=0$ and $\Lambda=0$. This is the functional analogue of basic properties of the quantum potential in the GQHJ theory that we now discuss.

The main point that characterizes the non-trivial properties of the quantum potential is its connection with the Möbius invariance of the Schwarzian derivative $\{f, x\}$, that, in order to be well-defined, requires that $f \in C^{2}(\mathbb{R})$ and $\partial_{x}^{2} f$ differentiable on $\mathbb{R}$. The continuity equation $\partial_{x}\left(R^{2} \partial_{x} S\right)=0$ implies that $R$ is proportional to $\left(\partial_{x} S\right)^{-1 / 2}$, so that the quantum potential can be expressed in terms of $S$ only

$Q=\frac{\hbar^{2}}{4 m}\{S, x\}$,

and the QHJE associated to a SSE reduces to the single equation

$\frac{1}{2 m}\left(\frac{\partial S}{\partial x}\right)^{2}+V-E+Q=0$.

Let us consider the basic identity

$$
\left(\frac{\partial S}{\partial x}\right)^{2}=\frac{\beta^{2}}{2}\left(\left\{\mathrm{e}^{\frac{2 i}{\beta} S}, x\right\}-\{S, x\}\right),
$$

where $\beta$ is a constant with the dimension of an action. Such an identity implies that the QHJE (3.6) can be also expressed in the form

$\left\{\exp \left(\frac{2 i}{\hbar} S\right), x\right\}=\frac{4 m^{2}}{\hbar}(E-V)$.

The solution of this non-linear differential equation is

$\exp \left(\frac{2 i}{\hbar} S\right)=\gamma\left[\frac{\psi^{D}}{\psi}\right]$

where $\psi$ and $\psi^{D}$ are two real linearly independent solutions of the SSE and $\gamma[f]$ is an arbitrary, generally complex, Möbius transformation of $f$

$\gamma[f]=\frac{A f+B}{C f+D}$.

Thanks to the Möbius invariance of the Schwarzian derivative, one may consider a Möbius transformation of $\exp (2 i S / \hbar)$, that we denote again by

$\gamma\left[\exp \left(\frac{2 i}{\hbar} S\right)\right]$,

leaving $V-E$ invariant. On the other hand, since this corresponds to the transformation

$S \longrightarrow \tilde{S}=\frac{\hbar}{2 i} \log \gamma\left[\exp \left(\frac{2 i}{\hbar} S\right)\right]$,

we see that there is a non-trivial mixing between the kinetic term and the quantum potential in (3.4).

In [5-10] the QHJE was derived by a slight modification of the way one gets the classical HJ equation. Namely, instead of looking for maps from $(x, p)$ to $(X, P)$, seen as independent variables, such that the new Hamiltonian is the trivial one, 
$\tilde{H}=0$, we looked for transformations $x \rightarrow \tilde{x}$ such that $\tilde{V}-$ $\tilde{E}=0$, but with the transformation of $p$ fixed by imposing that $S(x)$ transforms as a scalar function. We then have

$\tilde{S}(\tilde{x})=S(x)$,

holding for any pair of physical systems, including the one with $V-E=0$.

A key consequence of (3.11) is that $S(x)$ can never be a constant. In particular, imposing that (3.11) holds even when the coordinate $x$ refers to the state with $V-E=0$, forces the introduction of an additional term in the classical HJ equation. Then, one considers three arbitrary states, denoted by $A, B$ and $C$, and imposes the condition coming from the commutative diagram of maps

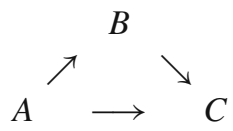

Implementation of such a consistency condition is equivalent to a cocycle condition that fixes the additional term to be the quantum potential [5-10]. The outcome is just the QHJE.

Another feature of the above formulation is that the quantum potential is never trivial even in the case $V-E=0$. In particular, a careful analysis of the quantum potential for a free particle with vanishing energy shows that the $\hbar \rightarrow 0$ and $E \rightarrow 0$ limits in the case of the free particle of energy $E$, leads to the appearance of the Planck length in the expression for the quantum potential $Q$ of a free particle with $E=0$, given in Eq. (1.1). It should be stressed that the present formulation leads to a well-defined power expansion in $\hbar$ for $S$. This is different with respect to the WKB approximation since $S_{\mathrm{WKB}}$ is defined by

$\psi=\exp \left(\frac{i}{\hbar} S_{\mathrm{WKB}}\right)$

so that, in general, $S_{\mathrm{WKB}}$ takes complex values. The GQHJ theory is also different with respect to the de Broglie-Bohm theory. Besides the case of real wave-functions illustrated above, also the quantum potential (1.1) turns out to be different. The difference also appears in the case of the free particle of energy $E$. Indeed, the solution of Eq. (3.4) with $V=0$ is

$S=\frac{\hbar}{2 i} \log \left(\frac{A e^{\frac{2 i}{\hbar} \sqrt{2 m E} x}+B}{C e^{\frac{2 i}{\hbar} \sqrt{2 m E} x}+D}\right)$.

Here the constants are chosen in such a way that $S \neq$ $\pm \sqrt{2 m E} x$. Such a choice, fixed by the consistency condition that the non-trivial $S_{E=0}$ is obtained from $S$ in the $E \rightarrow 0$ limit, relates $p$ - $x$ duality, also called Legendre duality, and Möbius invariance of the Schwarzian derivative [510]. Another consistency condition comes from the classical limit. Since $S^{c l}= \pm \sqrt{2 m E} x$, we have $\lim _{\hbar \longrightarrow 0} \log \left(\frac{A e^{\frac{2 i}{\hbar} \sqrt{2 m E} x}+B}{C e^{\frac{2 i}{\hbar} \sqrt{2 m E} x}+D}\right)^{\frac{\hbar}{2 i}}= \pm \sqrt{2 m E} x$,

implying that the constants $A, B, C$ and $D$ depend on $\hbar$ [5$10]$.

The above analysis shows that $S$ is the natural quantum analog of the classical Hamiltonian characteristic function. The formulation solves Einstein's paradox and the power expansion of $S$ in $\hbar$ is completely under control. Furthermore, it leads to a dependence of $S$ on the fundamental constants, shedding light on the quantum origin of interactions. It also implies that if space is compact, then time parametrization cannot be defined [12]. The formulation, that follows from the simple geometrical principle (3.11), extends to arbitrary dimensions and to the relativistic case as well [24]. It reproduces, together with other features, such as energy quantization, the non existence of trajectories, without assuming any interpretation of the wave-function.

\section{The WDW HJ equation with ${ }^{3} R=0$ and $\Lambda=0$}

Let us go back to the WDW equation by considering the case ${ }^{3} R=0, \Lambda=0$

$G_{i j k l} \frac{\delta^{2}}{\delta g_{i j} \delta g_{k l}} \Psi=0$.

Setting $\Psi=A e^{\frac{i}{\hbar} S}$, the WDW HJ equation reads

$G_{i j k l} \frac{\delta S}{\delta g_{i j}} \frac{\delta S}{\delta g_{k l}}-\frac{\hbar^{2}}{A} G_{i j k l} \frac{\delta^{2} A}{\delta g_{i j} \delta g_{k l}}=0$.

As shown in the previous section, a key difference between the GQHJ formulation and the Bohmian one, is that in the latter $R e^{\frac{i}{\hbar} S}$ is identified with the wave-function describing the physical state. This is not in general the case in the GCHJ formulation. In the Bohmian interpretation, the only admissible solution of Eq. (4.1) is the one where the wave-functional is trivial, so that, as in the case of the free particle with $E=0$, one would have $\Psi=0$, implying $A=0, S=0$ and $Q=0$. In the following we show that, as in the case of (1.1), the general solution of (4.1) implies non-trivial $A, S$ and $Q$.

Note that in the case of (4.1) the formulation does not suffer the well-known problem of the WDW equation, due to the presence of the second-order functional derivative at the same point: such an operator is in general ill-defined since it may lead to $\delta^{(3)}(0)$-singularities. On the other hand, the wave functional $\Psi\left[g_{i j}\right]$ now depends linearly on $g_{i j}$, so that the action of the second-order functional derivative on $\Psi\left[g_{i j}\right]$ is well-defined. We then have

$\Psi\left[g_{i j}\right]=A \mathrm{e}^{\frac{i}{\hbar} S}=\mathcal{T} g+C$, 
where

$\mathcal{T} g:=\int d^{3} \mathbf{x} \mathcal{T}^{j k}(\mathbf{x}) g_{j k}(\mathbf{x})$,

with $\mathcal{T}_{j k}(\mathbf{x})$ an arbitrary complex tensor density field of weight 1 and $C$ a complex constant. The most general expression of $S$ is

$\exp \left(\frac{2 i}{\hbar} S\right)=\frac{\mathcal{T} g+C}{\overline{\mathcal{T}} g+\bar{C}}$

and for $A$ we have

$A=|\mathcal{T} g+C|$

By (2.13) and (4.5), it follows that at the quantum level the momentum conjugate to $g_{j k}$ is

$\pi^{j k}=c \frac{\delta S}{\delta g_{j k}(\mathbf{x})}=\hbar c \operatorname{Im}\left(\frac{\mathcal{T}^{j k}(\mathbf{x})}{\mathcal{T} g+C}\right)$,

so that the kinetic term in the WDW HJ equation reads

$$
\begin{aligned}
2(c \kappa)^{2} G_{i j k l}(\mathbf{x}) \frac{\delta S}{\delta g_{i j}(\mathbf{x})} \frac{\delta S}{\delta g_{k l}(\mathbf{x})} \\
=\frac{2(c \kappa \hbar)^{2}}{\sqrt{g}}\left(\frac{\mathcal{T}_{k l}(\mathbf{x})}{\mathcal{T} g+C}\right) \operatorname{Im}\left(\frac{\mathcal{T}^{k l}(\mathbf{x})}{\mathcal{T} g+C}\right) \\
\left.\quad-\frac{1}{2}\left[\operatorname{Im}\left(\frac{\operatorname{Tr} \mathcal{T}(\mathbf{x})}{\mathcal{T} g+C}\right)\right]^{2}\right\} .
\end{aligned}
$$

Note that, by (4.2), this also corresponds to $-Q\left[g_{j k}\right]$. Furthermore, one may easily check that such an expression of $Q\left[g_{j k}\right]$ is just the functional analogue of the quantum potential of the free particle of vanishing energy (1.1).

\section{Cosmological constant from the quantum potential}

The discrepancy between the measured value of the cosmological constant and the theoretical prediction follows by considering $\Lambda / \kappa^{2}$ as a contribution to the effective vacuum energy density $\rho_{\text {eff }}=\rho+\Lambda / \kappa^{2}$, where $\left\langle T_{\mu \nu}\right\rangle=\rho g_{\mu \nu}$. Considering the QFT vacuum energy density as due to infinitely many zero-point energy of harmonic oscillators, we get (here $\hbar=c=1$ )

$\rho=\int_{0}^{\Lambda_{U V}} \frac{4 \pi k^{2} d k}{(2 \pi)^{3}} \frac{1}{2} \sqrt{k^{2}+m^{2}} \approx \frac{\Lambda_{U V}^{4}}{16 \pi^{2}} \approx 10^{71} \mathrm{GeV}^{4}$,

where $\Lambda_{U V}$ is the Planck mass. A result which is in complete disagreement with the estimation, based on experimental data, $\rho_{\text {eff }} \approx 10^{-47} \mathrm{GeV}^{4}$.

A problem with the above derivation is that it is based on the perturbative formulation of QFT. This corresponds to use the canonical commutation relations of the free theory that selects the vacuum of the free theory. On the other hand, the true vacuum of non-trivial QFT's is highly non-perturbative and is not unitarily equivalent to the free one. As a matter of fact, perturbation theory erroneously treats the quantum fields evolving as the free ones between point-like interaction events. From the physical point of view, the rôle of renormalization is to iteratively change the parameters of the theory, that then will depend on the physical scale. In other words, perturbation theory is a way to mimic the interacting theory by a free one, with the parameters becoming scale dependent.

It has been observed in [35] that the cutoff corresponding to the value of the cosmological constant may be related to an infrared/ultraviolet duality. In particular, the authors of [35], inspired by the Bekenstein bound $S \lesssim \pi M_{P}^{2} L^{2}$ for the total entropy in a volume of size $L^{3}$, proposed the following relation between the infrared cutoff $1 / L$ and $\Lambda_{U V}$

$L^{3} \Lambda_{U V}^{4} \lesssim L M_{P}^{2}$

An estimation of the infrared scale of QFT can be derived by considering the precision tests of the electron's anomalous magnetic moment $a_{e}$. In this respect, as observed in [36], an estimate of the correction to the usual calculation imposed by the IR scale $\mu$ is

$\delta a_{e} \approx \frac{\alpha}{\pi}\left(\frac{\mu}{m_{e}}\right) \approx 4 \times 10^{-9} \frac{\mu}{1 \mathrm{eV}}$

Requiring that such an indeterminacy be smaller than the uncertainty of the theoretical prediction for $a_{e}$ gives

$\mu \leq 10^{-2} \mathrm{eV}$

which is the value corresponding to the cutoff that leads to the same order of magnitude of the experimental value of $\rho$.

The above analysis indicates that the cosmological constant is related to the infrared problem, a non-perturbative phenomenon concerning the structure of the vacuum which has physically measured consequences. For example, QED finite transition amplitudes are obtained by summing over states with infinitely many soft photons.

We saw that, unlike in Bohmian mechanics, the quantum potential is never trivial [5-10]. This is the case even for the free particle of vanishing energy, implying that the quantum potential plays the rôle of particle intrinsic energy. Furthermore, Eq. (1.1) shows that the quantum potential includes the Planck length, which arises by consistency conditions in considering the $E \rightarrow 0$ and $\hbar \rightarrow 0$ limits [13]. This was one of the reasons suggesting a strict relationship between QM and GR [13] (see also [14]). We then have the following result:

The WDW quantum potential in the vacuum corresponds to an intrinsic energy density.

It is then natural to make the identification

$Q\left[g_{j k}\right]=-\sqrt{\bar{g}} \rho_{\mathrm{vac}}$, 
$\rho_{\text {vac }}=\Lambda / \kappa^{2}$. Since in this case the only degrees of freedom are the ones associated to the metric tensor, the dark energy should correspond to a graviton condensate.

In this context, we stress that the vacuum energy is a purely quantum property and the absence of the kinetic term does not imply, as in the de Broglie-Bohm theory, Einstein's paradox. The fact that the cosmological constant is a quantum correction to the Einstein tensor given in terms of the quantum potential, is reminiscent of the von Weizsäcker correction to the kinetic term of the Thomas-Fermi theory. Furthermore, we note that the quantum potential also defines the Madelung pressure tensor.

Now observe that the absence of propagating degrees of freedom implies that the quantum potential in (5.5) corresponds to the one of the WDW HJ equation without the kinetic term, that is

$S=0$.

Let us choose a metric with vanishing ${ }^{3} R$. Equation (5.6) implies a nice mechanism, namely by (2.18) it follows that in this case the continuity equation is trivially satisfied, so that Eq. (5.5), that by (5.6) is the full WDW HJ equation, coincides with the WDW equation (2.15) with $\Psi=A$. In this way the contribution to the WDW HJ equation comes only from the quantum potential. In other words, since by (5.6) $\Psi$ takes real values, it follows by the definition of $Q\left[g_{i j}\right]$ in (2.20), that Eq. (5.5) is just the WDW equation in the vacuum

$$
-2 \ell_{P}^{2} G_{i j k l} \frac{\delta^{2}}{\delta g_{i j} \delta g_{k l}} A=-\frac{\sqrt{\bar{g}}}{\ell_{P}^{2}} \Lambda A .
$$

Note that such an equation is just the functional analog of a stationary Schrödinger equation with negative energy. This suggests considering the role of fundamental scales. To this end we adapt the analysis that led to Eq. (1.1), to the case of Eq. (5.7). The main difference is that now the problem includes both small and large scales. To see how fundamental constants may appear in the present context, we first derive an explicit solution of Eq. (5.7) in the case of the FriedmannLemaître-Robertson-Walker background.

Let us then consider the line element

$d s^{2}=-N(t)^{2} c^{2} d t^{2}+a^{2}(t) d \Sigma_{k}^{2}$,

where

$d \Sigma_{k}^{2}=\frac{d r^{2}}{1-k r^{2}}+r^{2}\left(d \theta^{2}+\sin ^{2} \theta d \phi^{2}\right)$,

is the spatial line element of constant curvature $k$. In such an approximation the Hilbert-Einstein equation in the vacuum, with $k=0$, reads

$S_{H E}=\frac{V_{0}}{\kappa^{2}} \int d t\left(-\frac{3 a \dot{a}^{2}}{N c}-N c a^{3} \Lambda\right)$, where

$V_{0}=\int d r d \theta d \phi r^{2} \sin \theta$

In such a minisuperspace approximation, the WDW equation (5.7) reads

$$
\left(\frac{d^{2}}{d a^{2}}+12 \frac{V_{0}^{2} \Lambda}{\ell_{P}^{4}} a^{4}\right) A_{F L R W}=0,
$$

whose solution is a linear combination of the Bessel functions of first and second kind

$A_{F L R W}(a)=\sqrt{a}\left(\alpha J_{1 / 6}\left(C a^{3}\right)+\beta Y_{1 / 6}\left(C a^{3}\right)\right)$,

where

$C=2 \frac{V_{0}}{\ell_{P}^{2}} \sqrt{\frac{\Lambda}{3}}$.

In this approximation of the WDW equation, besides the Planck length, there is also another fundamental constant, $\Lambda$ itself, and a natural choice, suggested by (5.14), would be

$V_{0}=\Lambda^{-3 / 2}$.

Such a result provides an indication on the possible appearance of scales related to the WDW equation. Nevertheless, the analysis should be done in the framework of the original WDW equation, not just considering its minisuperspace approximation. A key aspect is that the WDWW equation is ill-defined, in particular it must be regularized, a problem which is completely missing in the minisuperspace approximation. In the following, we will see that a fundamental scale may in fact appear as an infrared regulator. In agreement with Dirac's idea, we then will suggest that fundamental constants may be dynamical variables.

\section{Infrared/ultraviolet duality and local to global geometry theorems}

In this section we make some speculation concerning the infrared/ultraviolet duality in the context of the WDW equation, which is the natural framework to investigate the relations between the structure of the Universe and small scales. We saw that such an equation includes both large and small scales that can be interpreted as infrared and ultraviolet cutoffs, that should appear in a well-defined version of the WDW equation. It is clear that such an investigation should include a careful analysis of the involved local and global geometries.

A well-known problem with the WDW equation, is that due to the second-order functional derivative evaluated at the same point, it presents, in general, $\delta^{(3)}(\mathbf{x}=\mathbf{0})$-singularities. This is analogous to the normal ordering singularities in QFT, due to the joining of two legs of the same vertex; so giving the Feynman propagator evaluated at 0 . Similarly, the infinite 
volume limit can be interpreted as the integral representation of the $\delta$-distribution in momentum space at zero momentum. In other words, $\delta^{(3)}(\mathbf{p}=\mathbf{0})$ can be interpreted as the infinite space volume limit divided by $(2 \pi)^{3}$. A related method is used, for example, in deriving the effective action for $\lambda \phi_{4}^{4}$ in Euclidean space to get the dependence of the coupling constant on the mass scale. In that case, the infrared regularization was done by supposing that the Euclidean space is $S^{4}$ rather than $\mathbb{R}^{4}$, and then considering $S^{4}$ as the surface of a five-dimensional sphere, so that one obtains a finite result and avoids such an infrared divergence.

The outcome of such an analysis is that in general singularities may be removed by taking into account the physical scales. What is crucial is to preserve diffeomorphism invariance. In this respect, we recall that $\left[-i \hbar \delta_{g_{j k}}\right]=M L^{-1} T^{-1}$, while for the $\delta$-distribution in configuration space we have $\left[\delta^{(3)}\right]=L^{-3}$. This means that, besides the Planck length, a well-defined regularized version of the WDW equation should also involve a large scale cutoff. An explicit example of such a mechanism is the one in the interesting paper by Feng, who proposed the volume average regularization [30]. Feng's regularization introduces a factor $1 / V$, with $V$ naturally identified with the space volume. In particular, Feng's regularized WDW equation has the structure

$\hat{\mathcal{H}}\left[\ell_{P}, V, \Lambda ; g_{i j}\right] \Psi\left[g_{i j}\right]=0$,

see, for example, Eq. (2.24) of [30]. Feng's regularization is related to the standard heat kernel and point splitting regularizations [37-41]. In particular, it corresponds to averaging the displacement in the point splitting regularization.

The dual rôle of the $\delta$-distributions in $x$ and $p$ spaces, shows that infrared and ultraviolet dualities are related to $x-p$ duality, another manifestation of the dual property of the Fourier transform, which in fact is at the heart of the Heisenberg uncertainty relations.

Even if a well-defined version of the WDW functional differential equation is still unknown, it is clear that, as Eq. (5.12) shows, besides the Planck length it should also include a cosmological scale, making manifest an infrared/ultraviolet duality. A related issue concerns the Möbius symmetry of the Schwarzian derivative. In this respect, it was shown in [24] that even in the geometrical derivation of the QHJE in higher dimensions, there is an underlying global conformal symmetry, the generalization of the Möbius symmetry of the Schwarzian derivative. This is a crucial property, whose implementation requires a compact space, which in turn would imply that the energy spectra are quantized [12]. As a consequence, since by Jacobi theorem [15-23]

$t-t_{0}=\frac{\partial S}{\partial E}$ it follows that time-parametrization is ill-defined for discrete spectra, so that trajectories would never exist if space is compact [12]. The mentioned conformal transformation includes the space inversion relating large and small scales

$x_{k} \rightarrow l^{2} x_{k} / r^{2}$

$r^{2}=\sum_{1}^{D} x_{k}^{2}$, with $l$ a length scale. This is another hint that an infrared/ultraviolet duality should appear in the cosmological context, and then in a well-defined version of the WDW equation. A similar situation arises in the uniformization theory by Klein, Koebe and Poincaré, where negatively curved Riemann surfaces have fundamental domains in their universal covering, e.g. the upper half-plane $\mathbb{H}$, which are related by Fuchsian transformations, that is discrete subgroups of ${ }^{2}$ $\operatorname{SL}(2, \mathbb{R})$.

Finding an infrared/ultraviolet duality in the cosmological context could be used to consider the local to global theorems relating local and global geometries. In particular, according to Thurston [25], the global geometry is strongly constrained in case the local one has constant curvature. Interestingly, according to Bieberbach $[42,43]$, all compact flat manifolds are finitely covered by tori, a result that in three dimension was previously obtained by Schoenflies [44]. The underlying idea is that the local structure of space provides information on its global structure, which includes the information on the topological structure and on points at large distances.

The discussed connection between compactness of space, discrete spectra and the analogies with uniformization theory, suggests that higher dimensional uniformization theory is the right framework to investigate the geometry of the universe.

It is clear that the solution of a well-defined version of the WDW equation should involve transcendental functions; a property which already appears in the minisuperspace approximation. As such, the dependence on the cosmological constant should be in the form of some dimensionless constant $\mathcal{K}$, that is

$A\left[g_{i j}\right]=F\left[\mathcal{K} ; g_{i j}\right]$.

Note that $\mathcal{K}$ should be the same for any choice of the time slicing in the ADM foliation, so that $\mathcal{K}$ should be timeindependent. Since the Planck length is naturally interpreted as ultraviolet cutoff, we have

$\mathcal{K}=\frac{\ell_{P}}{L_{U}}$,

with $L_{U}$ a fundamental length describing the geometry of the Universe. The obvious candidate for $L_{U}$ is the Hubble radius $R_{H}=c / H_{0}=1.36 \times 10^{26} \mathrm{~m}$, whose size is of the

\footnotetext{
2 This is in fact deeply related to the weak/strong duality transformations of the effective coupling constant $\tau \rightarrow-1 / \tau$ of Seiberg-Witten theory, that, in the case of pure $\mathrm{SU}(2)$, posses a $\Gamma(2) \subset \mathrm{SL}(2, \mathbb{R})$ symmetry.
} 
same order of the radius of the observable universe and that, besides $\Lambda$, is the only quantity which is spatially constant. We then have,

$\mathcal{K}=\frac{\ell_{P}}{R_{H}}=5.96 \times 10^{-61}$.

Furthermore, since $A$ must depend on $\Lambda$, the space-time independence of $\mathcal{K}$ implies that

$A\left[g_{i j}\right]=F\left[D \sqrt{\Lambda} ; g_{i j}\right]$,

with $D,[D]=L$, a space-time constant.

Equation (6.6) would imply that the Planck length is timedependent. This is in agreement with the Dirac idea that fundamental constants are dynamical variables. On the other hand, the most natural candidate for time variation is just the Planck constant $\hbar$. The point is that the Einstein field equation contains $\Lambda, c$ and $G$, and a possible time dependence of such constants would break diffeomorphism invariance. Therefore, preserving such an invariance means that only $\hbar$, that in fact appears only in considering the WDW equation, can change. On the other hand, Eq. (6.6) implies an infrared/ultraviolet duality, where the large scale is given by $R_{H}$, whose time dependence is the same of the scale representing the quantum regime, that is (the square root of) $\hbar$.

We stress that time variation of fundamental constants is a crucial and widely investigated subject [45-47]. In a different context, time dependence of the Planck constant has been investigated in the interesting paper [48].

We conclude by observing that very recently, in [49], it has been argued by a different perspective, that the GQHJ theory introduced in [5-10], could in fact be at the origin of the cosmological constant.

Acknowledgements It is a pleasure to thank N. Bartolo, D. Bertacca, K. Lechner, S. Matarrese, M. Peloso and A. Ricciardone for interesting comments and discussions.

Data Availability Statement This manuscript has no associated data or the data will not be deposited. [Authors' comment: Data sharing not applicable to this article as no datasets were generated or analyzed during the current study.]

Open Access This article is licensed under a Creative Commons Attribution 4.0 International License, which permits use, sharing, adaptation, distribution and reproduction in any medium or format, as long as you give appropriate credit to the original author(s) and the source, provide a link to the Creative Commons licence, and indicate if changes were made. The images or other third party material in this article are included in the article's Creative Commons licence, unless indicated otherwise in a credit line to the material. If material is not included in the article's Creative Commons licence and your intended use is not permitted by statutory regulation or exceeds the permitted use, you will need to obtain permission directly from the copyright holder. To view a copy of this licence, visit http://creativecomm ons.org/licenses/by/4.0/.

Funded by SCOAP ${ }^{3}$.

\section{References}

1. S. Weinberg, The cosmological constant problem. Rev. Mod. Phys 61, 1-23 (1989)

2. S.M. Carroll, The cosmological constant. Living Rev. Relativ. 4, 1 (2001). arXiv:astro-ph/0004075

3. J. Sola, Cosmological constant and vacuum energy: old and new ideas. J. Phys. Conf. Ser. 453, 012015 (2013). arXiv:1306.1527

4. A.E. Faraggi, M. Matone, Duality of $x$ and $\psi$ and a statistical interpretation of space in quantum mechanics. Phys. Rev. Lett. 78, 163 (1997). arXiv:hep-th/9606063

5. A.E. Faraggi, M. Matone, Quantum mechanics from an equivalence principle. Phys. Lett. B 450, 34-40 (1999). arXiv:hep-th/9705108

6. A.E. Faraggi, M. Matone, The equivalence principle of quantum mechanics: uniqueness theorem. Phys. Lett. B 437, 369-380 (1998). arXiv:hep-th/9711028

7. A.E. Faraggi, M. Matone, Quantum transformations. Phys. Lett. A 249, 180-190 (1998). arXiv:hep-th/9801033

8. A.E. Faraggi, M. Matone, Equivalence principle, Planck length and quantum Hamilton-Jacobi equation. Phys. Lett. B 445, 77-81 (1998). arXiv:hep-th/9809125

9. A.E. Faraggi, M. Matone, Equivalence principle: tunneling, quantized spectra and trajectories from the quantum $\mathrm{HJ}$ equation. Phys. Lett. B 445, 357-365 (1999). arXiv:hep-th/9809126

10. A.E. Faraggi, M. Matone, The equivalence postulate of quantum mechanics. Int. J. Mod. Phys. A 15, 1869-2017 (2000). arXiv:hep-th/9809127

11. A.E. Faraggi, M. Matone, The equivalence postulate of quantum mechanics: main theorems. Quantum Trajectories, ed. P. Chattaraj, Taylor \& Francis/CRC Press (2011) Print ISBN: 978-1-4398-25617. eBook ISBN: 978-1-4398-2562-4. arXiv:0912.1225 [hep-th]

12. A.E. Faraggi, M. Matone, Energy quantisation and time parameterisation. Eur. Phys. J. C 74, 2694 (2014). arXiv:1211.0798 [hep-th]

13. M. Matone, Equivalence postulate and quantum origin of gravitation. Found. Phys. Lett. 15, 311 (2002). arXiv:hep-th/0005274

14. L. Susskind, Dear qubitzers, GR $=$ QM. arXiv:1708.03040 [hep-th]

15. E.R. Floyd, Phys. Rev. D 25, 1547 (1982)

16. E.R. Floyd, Phys. Rev. D 26, 1339 (1982)

17. E.R. Floyd, Phys. Rev. D 29, 1842 (1984)

18. E.R. Floyd, Phys. Rev. D 34, 3246 (1986)

19. E.R. Floyd, Found. Phys. Lett. 9, 489 (1996)

20. E.R. Floyd, Found. Phys. Lett. 13, 235 (2000)

21. E.R. Floyd, Phys. Lett. A 214, 259 (1996)

22. E.R. Floyd, Int. J. Mod. Phys. A 14, 1111 (1999)

23. E.R. Floyd, Int. J. Mod. Phys. 15, 1363 (2000)

24. G. Bertoldi, A.E. Faraggi, M. Matone, Equivalence principle, higher dimensional Mobius group and the hidden antisymmetric tensor of quantum mechanics. Class. Quantum Gravity 17, 3965 (2000). arXiv:hep-th/9909201

25. W. Thurston, in Three-Dimensional Geometry and Topology, vol. 1, Princeton Mathematical Series, vol. 35, ed. by S. Levy (Princeton University Press, Princeton, 1997)

26. B.S. DeWitt, Quantum theory of gravity. 1. The canonical theory. Phys. Rev. 160, 1113 (1967)

27. J.A. Wheeler, Superspace and the nature of quantum geometrodynamics, in Battelle Rencontres, ed. by C.M. DeWitt, J.A. Wheeler (Benjamin, New York, 1968), p. 242

28. C.F. Weizsäcker, Zur Theorie der Kernmassen. Zeitschrift für Physik 96, 431 (1935)

29. S. Das, R.K. Bhaduri, Dark matter and dark energy from a BoseEinstein condensate. Class. Quantum Gravity 32(10), 105003 (2015). arXiv:1411.0753 [gr-qc]

30. J.C. Feng, Volume average regularization for the Wheeler-DeWitt equation. Phys. Rev. D 98(2), 026024 (2018). arXiv:1802.08576 [gr-qc] 
31. R.L. Arnowitt, S. Deser, C.W. Misner, The dynamics of general relativity. Gen. Relativ. Gravit. 40, 1997-2027 (2008). https://doi. org/10.1007/s10714-008-0661-1. arXiv:gr-qc/0405109 [gr-qc]

32. M. Blau, Lecture Notes on General Relativity. http://www.blau.itp. unibe.ch/newlecturesGR.pdf

33. G. Calcagni, Classical and Quantum Cosmology (Springer, Cham, 2017)

34. P.R. Holland, The Quantum Theory of Motion (Cambridge University Press, Cambridge, 1993)

35. A.G. Cohen, D.B. Kaplan, A.E. Nelson, Effective field theory, black holes, and the cosmological constant. Phys. Rev. Lett. 82, 49714974 (1999). arXiv:hep-th/9803132

36. J.M. Carmona, J.L. Cortes, Infrared and ultraviolet cutoffs of quantum field theory. Phys. Rev. D 65, 025006 (2002). arXiv:hep-th/0012028

37. R.M. Wald, Trace anomaly of a conformally invariant quantum field in curved space-time. Phys. Rev. D 17, 1477-1484 (1978)

38. P. Mansfield, Continuum strong coupling expansion of Yang-Mills theory: quark confinement and infrared slavery. Nucl. Phys. B 418, 113-130 (1994). arXiv:hep-th/9308116 [hep-th]

39. T. Horiguchi, K. Maeda, M. Sakamoto, Analysis of the WheelerDeWitt equation beyond Planck scale and dimensional reduction. Phys. Lett. B 344, 105-109 (1995). arXiv:hep-th/9409152

40. K. Maeda, M. Sakamoto, Strong coupling quantum gravity and physics beyond the Planck scale. Phys. Rev. D 54, 1500-1513 (1996). arXiv:hep-th/9604150
41. J. Kowalski-Glikman, K.A. Meissner, Phys. Lett. B 376, 48-52 (1996). arXiv:hep-th/9601062

42. L. Bieberbach, Über die Bewegungsgruppen der Euklidischen Räume I. Math. Ann. 70(3), 297-336 (1911)

43. L. Bieberbach, Über die Bewegungsgruppen der Euklidischen Räume II: Die Gruppen mit einem endlichen Fundamentalbereich. Math. Ann. 72(3), 400-412 (1912)

44. A. Schoenflies, Kristallsysteme und Kristallstruktur (Teubner, Stuttgart, 1891)

45. J.P. Uzan, Rev. Mod. Phys. 75, 403 (2003). arXiv:hep-ph/0205340

46. J.P. Uzan, Living Rev. Relativ. 14, 2 (2011). arXiv:1009.5514 [astro-ph.CO]

47. L. Hart, J. Chluba, New constraints on time-dependent variations of fundamental constants using Planck data. Mon. Not. R. Astron. Soc. 474(2), 1850-1861 (2018). arXiv:1705.03925 [astro-ph.CO]

48. G. Mangano, F. Lizzi, A. Porzio, Inconstant Planck's constant. Int. J. Mod. Phys. A 30(34), 1550209 (2015). arXiv:1509.02107 [quant-ph]

49. J. Ben Achour, E.R. Livine, The Cosmological Constant from Conformal Transformations: Möbius Invariance and Schwarzian Action. Class. Quant. Grav. 37(21), 215001 (2020). https://doi. org/10.1088/1361-6382/abb577. arXiv:2004.05841 [gr-qc] 